\title{
GOBERNANZA DE LAS NANOTECNOLOGÍAS
}

\author{
Javier Echeverría \\ Departamento de Ciencia, Tecnologia y Sociedad \\ Instituto de Filosofia, CSIC
}

\begin{abstract}
Nanotechnologies and the Converging Technologies Programmes (CT) put forward relevant challenges to the governance of science and technology, because their development takes place in the context of a transnational $R+D+i$ system with a plurality of competitive and cooperative agents who pursue a diversity of goals. How to integrate the plurality of technoscientific agencies and how to establish the research priorities of CT Programmes constitute significant problems. This paper presents a comparison between the American (NBIC) and European (CTEKS) CT programmes in order to analyse their commonalities and differences. It is argued that nanosciences and nanotechnologies constitute one of the main instances of contemporary technoscience, assuming the thesis that in technoscience (contrary to what happens in traditional science), scientific knowledge is not and end in itself, but just an instrument to obtain social, political, economic or military ends. Then, it is proposed a general philosophical hypotesis about the colonization and industrialization of the nanocosmos, and the notion of scientific agenda is put forward in order to analyse and characterise the diverse models of governance.
\end{abstract}

KEY WORDS Governance of science and technology, nanosciences, nanotechnologies, Converging Technologies, scientific agenda.
RESUMEN Las nanotecnologías y los programas Converging Technologies (CT) plantean importantes desafíos a la gobernanza de la ciencia y la tecnología, al desarrollarse en un sistema transnacional de $I+D+i$ con una pluralidad de agentes que compiten y cooperan entre si, y que tienen intereses y objetivos diversos. Integrar esa pluralidad de agencias tecnocientíficas y fijar las prioridades de los diversos programas CT es el principal problema. Tras comparar los programas norteamericano (NBIC) y europeo (CTEKS), este artículo analiza sus principales puntos comunes, pero también sus diferencias, que son significativas. Se afirma que las nanociencias y las nanotecnologías constituyen uno de los grandes ejemplos de tecnociencia contemporánea, y se distingue la tecnociencia de la ciencia porque en el primer caso el conocimiento científico es un medio para el logro de otros objetivos (sociales, políticos, económicos, militares, etc.), no un fin en sí mismo. Además de proponer una hipótesis filosófica general, la colonización e industrialización de los nanocosmos, se utiliza la noción de agenda científica como herramienta para analizar y caracterizar los diversos modelos de gobernanza actualmente existentes.

PALABRAS CLAVE Gobernanza de la ciencia y la tecnologia, nanociencias, nanotecnologías, Converging Technologies, agenda científi-

\section{Introducción ${ }^{1}$}

Las nanociencias (NC) y las nanotecnologias (NT) constituyen un excelente caso de estudio para analizar los principales problemas de la gobernanza de las ciencias y las tecnologías en los actuales sistemas de $1+D+i^{2}$. En primer lugar, porque han generado grandes expectativas ${ }^{3}$ y no pocos avances cientificos y tecnológicos. En segundo lugar, porque han suscitado diversos problemas y riesgos. En tercer lugar, porque afectan a numerosas disciplinas, ponen en cuestión la separación tradicional entre ciencia y tecnología y trastocan las fronteras académicas y profesionales, generando equipos y centros de investigación multidisciplinares e interinstitucionales ${ }^{4}$. En cuarto lugar, se requieren grandes inversiones, la colaboración activa de empresas e industrias y la aceptación social de la nueva mainstream cientifica. Las iniciativas y avances en los campos NC y NT provienen de diversas agencias tecnocientificas que compiten y cooperan entre si en un sistema multiagente. Por tanto, es precisa una legislación específica para el sector, pues los problemas jurídicos que plantean las innovaciones NT generadas por esas agencias y consorcios son importantes. Estos cuatro factores plantean otros tantos desafios a la gobernanza de la ciencia.

Un quinto aspecto a considerar es la competencia internacional que la investigación NC y NT suscita, así como los problemas que se derivan de la apropiación de ese tipo de conocimiento. No estamos ante un sistema nacional de $1+D+i$ que fomenta el conocimiento cientifico por el bien del pais $y$, por asi decirlo, "nacionaliza" el conocimiento, sino ante una confluencia de varios sistemas que compiten y 
cooperan en el campo NT, compartiendo el conocimiento, pero también apropiándoselo.

Siendo el término "gobernanza" relativamente impreciso y polivoco, partiremos de la definición de gobernanza que Emilio Muñoz ha propuesto recientemente: «es la puesta en práctica de formas de gobierno estratégicas para poner de relieve el valor de lo público a través de la relación entre sociedad, mercado y Estado y conseguir de este modo un desarrollo socialmente sosteniblen ${ }^{5}$. Asimismo asumiremos la hipótesis de que las NC y las NT no sólo son ciencias y tecnologías, sino algo más, a saber: un híbrido entre ambas, la tecnociencia, que ha ido surgiendo y consolidándose a lo largo del siglo XX6. No es lo mismo la gobernanza de la ciencia que la de la tecnociencia, porque ésta última implica una pluralidad de agentes con valores e intereses diversos, que hay que armonizar y hacer confluir. Los macroprogramas Converging Technologies (CT) de los EEUU y la UE ${ }^{7}$ son típicamente tecnocientíficos. Por esta razón hablaremos de nanotecnociencias (NTC), más que de nanociencias (NC) y nanotecnologías (NT).

Las NTC traen consigo problemas especificos, entre los cuales consideraremos los cinco siguientes:

- Pluralidad de agentes tecnocientíficos que intervienen en el actual impulso a las NTC: empresarios, políticos, investigadores, tecnólogos, militares, expertos en gestión e innovación, juristas, publicistas, representantes de la sociedad civil, etc.

- Existencia de estrategias diferentes para impulsar las NTC según los diversos países, como queda claro en los macroprogramas CT de los EEUU y la UE. Compararemos y contrapondremos ambas estrategias.

- Relaciones entre las NTC y la sociedad: percepción social, oportunidades y riesgos, control social, NTC-ficción, propiedad intelectual, patentes, legislación, recepción social de las innovaciones NTC (mercado), participación social en los programas $\mathrm{CT}$, etc.

- Incidencia de las NTC en el desarrollo social sostenible, en la medida en que pueden contribuir a solucionar, paliar 0 agudizar algunos de los principales problemas que tienen planteadas las sociedades contemporáneas: energía, nutrición, sanidad, cambio climático, brecha tecnológica y emergencia de las sociedades de la información y el conocimiento.

- Transformación de los sistemas educativos y formativos de científicos y expertos NTC, que puede traer consigo profundos cambios en la estructura académica (Departamentos e Institutos mixtos, cambios en los planes de estudio, formación continua, fomento de la actitud innovadora en los procesos de aprendizaje, etc.). Este es uno de los aspectos más significativos de la gobernanza de las NTC.

En este artículo vamos a centrarnos en las dos primeras cuestiones, dejando las restantes para contribuciones ulteriores. Previamente, propondremos un marco filosófico para analizar las nanotecnologías, basado en la tesis de que las NTC implican el descubrimiento de un nuevo mundo, los nanocosmos y nanosistemas, que ya existían en la naturaleza pero ahora empiezan a ser explorados por los tecnocientíficos. Esta será la primera propuesta de este artículo, por una parte ontológica, pero también políticosocial. Partiendo de este marco conceptual, abordaremos los dos aspectos recién mencionados de la gobernanza de las NTC.

\section{Breve cronología de la emergencia de las nanotecnociencias (NTC)}

La investigación científica a escala nanométrica es anterior a los diversos programas CT. Conviene recordar brevemente la etapa estrictamente científica (1980-2000) para distinguirla de la tecnocientífica, que comienza en el año 2000, fecha en la que el Presidente Clinton anunció la National Nanotechnology Initiative (NNI). Lo haremos muy sucintamente:

1959: Richard Feynman, Premio Nobel de Física, pronuncia una célebre conferencia, "There's plenty of room at the bottom", en la que concibe la posibilidad de una ingenieria a nivel atómico, requiriéndose para ello las herramientas adecuadas, no disponibles en aquel tiempo ${ }^{8}$.

1974: Norio Taniguchi (Universidad de Ciencias de Tokyo) introduce el término "nanotecnologia»".

1981: Gerd K. Binnig y Heinrich Rohrer (ulteriormente Premios Nobel de Fisica) inventan en el Laboratorio de Investigación de IBM en Zürich el microscopio de efecto túnel (scanning tunneling microscope, STM) que permitió por primera vez a los investigadores representar los átomos. Este nuevo artefacto tecnológico ha desempeñado un papel fundamental en la investigación ulterior. 
1985: Robert F. Curl, jr., Harond W. Croto y Richard E. Smalley descubren las buckyballs, que miden aproximadamente un nanómetro. En 1996 se les concedió el Premio Nobel de Quimica.

1986: Eric Drexler abre la vía de investigación "bottom-up», al proponer construir objetos a partir de sus componentes atómicos y moleculares. Asimismo abre el frente de la nanocienciaficción, al imaginar robots que podrian autoensamblarse ${ }^{10}$.

1989: Los físicos comienzan a manipular átomos con precisión, deletreando letras con átomos xenon 35 .

1991: Sumio ljima (NEC Research Laboratories, Japón) descubre los nanotubos de carbón, el segundo gran avance tecnológico en el campo NTC.

1992: Comienzan a utilizarse los microscopios de fuerzas atómicas, que han conformado una vía alternativa a los microscopios de efecto túnel (STM).

1993: Warren Roninet (University of North Carolina) y R. Stanley Williams (University of California) crean un sistema de realidad virtual conectado a un STM que permite a los científicos "ver" y "tocar" átomos.

1993: La Rice University (USA) crea el primer laboratorio dedicado a las nanotecnologías.

1996: Investigadores de la Delft University of Technology (Holanda) crean un transistor usando nanotubos de carbón. Se abre la via de convergencia entre las NTC y las tecnologias de la información y comunicación (TIC).

1996: Varias agencias federales norteamericanas promueven el plan NNI (National Nanotechnology Initiative) y comienzan a crear Centros de Investigación sobre nanotecnologias: catorce entre 1996 y 2003. Otro tanto ocurre en el sector privado. Estas agencias tecnocientíficas comienzan a demandar programas federales para impulsar la investigación en el sector.

1998: El Ministerio alemán de Investigación y Tecnología crea seis centros nacionales dedicados a las nanotecnologias. Otros paises (Francia, Reino Unido, Japón, etc.) hacen otro tanto.

1998: La Unión Europea lanza la iniciativa NID (Nanotechnology Information Devices) dentro del plan IST (Informa- tion Society Technologies). Las NTC muestran su utilidad para mejorar las TIC, conforme a la tendencia a la miniaturización de los chips.

1999: El Departamento norteamericano de Defensa designa a las nanotecnologías como un área de importancia estratégica.

2000: Lucent \& Bell Laboratories, en colaboración con la Universidad de Oxford, crean el primer motor de DNA, demostrando la posibilidad de convergencia entre las biotecnologías y las nanotecnologías.

2001: La National Science Foundation (NSF) norteamericana hace público su plan Converging Technologies for Improving Human Performance, habitualmente denominado NBIC (nano-bio-info-cogno), asi como su informe sobre las implicaciones sociales de dicho plan.

2002: El Canadian National Research Council promueve asimismo su Plan Converging Technologies for bio-health, eco and food system integrity and disease mitigation (2003) ${ }^{11}$.

2003: La Unión Europea crea un grupo de expertos, que presenta en junio de 2004 el programa europeo Converging Technologies for the European Knowledge Society (CTEKS).

Asimismo publica un primer informe sobre riesgos posibles.

Podrian evocarse otros muchos hitos, pero los anteriores bastan para ilustrar dos de las caracteristicas principales de la investigación en el sector NTC:

1. Los principales avances han sido tecnológicos e instrumentales, al irse inventando diferentes tipos de microscopios que han hecho accesible la materia a escala nanométrica, tanto para observarla como para operar con ella. La principal especificidad de las nanotecnologias consiste en la capacidad de manipular los átomos, las moléculas, el ribosoma, el DNA y las células con el fin de modificar sus propiedades y su estructura interna. Las nanotecnologías transforman la materia a nivel atómico, posibilitando la creación de nuevos materiales, orgánicos e inorgánicos. Por supuesto, a lo largo de toda esta etapa se fue generando mucho conocimiento científico, y muy relevante. Pero el paso de las nanociencias NC a las nanotecnociencias NTC se produjo conforme diversos instrumentos abrieron la posibilidad de que los seres humanos intervengan y operen a escala nanométrica. 
2. Paralelamente a esos avances, comenzaron a crearse centros de investigación especializados, tanto públicos como privados. La investigación estrictamente científica y tecnológica que hasta entonces se había llevado a cabo, realizando el proyecto visionario de Feynman, dio paso a la actividad tecnocientifica propiamente dicha, que tiene caracteristicas muy distintas. Todo ello confluyó en diversos programas CT (EEUU, Canadá, UE, etc.), que comienzan a ser formulados e implementados a partir del año 2000. La existencia de esos macroprogramas de investigación marca la emergencia de la nanotecnologia como tecnociencia vinculada a las TIC, las biotecnologias, las ciencias cognitivas y otras disciplinas científicas. Es importante subrayar que todos los paises cientificamente desarrollados han elaborado planes de fomento de las NTC. Este es el aspecto de la gobernanza que pretendemos analizar.

\section{El descubrimiento de los nanosistemas y los nanocosmos}

Como acabamos de indicar, en una conferencia seminal pronunciada en 1959, el físico Richard Feynman sostuvo una tesis visionaria: "there's plenty of room at the bottom»" Indicaba asi la existencia de un nuevo ámbito a investigar: la materia y la energía a escala nanométrica. Buena parte de la investigación física en aquella época se orientaba a lo macrocósmico, singularmente a la exploración del espacio exterior al planeta Tierra. Diversos microcosmos, orgánicos e inorgánicos, habian sido investigados por físicos, quimicos y biólogos desde la emergencia de la ciencia moderna, gracias a una gran variedad de instrumentos científicos que permitieron determinar y medir las propiedades de la materia y la energía a escala meso- y microcósmica. Los telescopios y microscopios han sido dos de los cánones de la cultura científica experimental desde los siglos XVI y XVII. Pues bien, Feynmann advertía que, si se llegaban a construir aparatos para investigar el mundo físico a escala nanométrica (10-9 $\mathrm{mm})$, quedaba todo un mundo por descubrir y explorar. Para ello era preciso poder observarlo $y$, de ser posible, habia que construir aparatos que permitieran a los físicos operar y manipular la materia a escala molecular y atómica.

Este objetivo se logró un cuarto de siglo después, gracias al microscopio de efecto túnel, diseñado por Bibbig y Rohrer en 1981-82. Aunque con diversas limitaciones, dicho microscopio proporcionaba una topografía tridimensional de superficies a nivel atómico, es decir, una primera representación científica de la materia a escala nanométrica. Los átomos no se veían, no aparecian, pero sí podian ser representados mediante imágenes tridimensionales. Tras la invención del microscopio de efecto túnel (scanning tunneling microscope, STM), por la que Bibbig y Rohrer obtuvieron el premio Nobel en 1986, se sucedieron las mejoras tecnológicas y fueron apareciendo nuevos microscopios que permitían obtener representaciones cada vez más precisas de la materia a nivel atómico ${ }^{13}$.

Esta línea de investigación, todavía reciente, tiene gran interés para la teoria representacional de la ciencia ${ }^{14}$, para la ontología de la ciencia (dualidad onda-corpúsculo de la mecánica cuántica) y para la filosofía de la tecnociencia, puesto que inaugura una nueva capacidad humana, la de operar tecnocientíficamente a escala atómica. Nos centraremos en esta última cuestión, que es la que permite hablar de la colonización de los nanocosmos y suscita el problema de cómo va a llevarse a cabo su industrialización. A nuestro juicio, éste es el principal desafío para la gobernanza de las NTC ${ }^{15}$.

Gracias a estos nanoscopios, como deberían ser denominados, la ingeniería ha empezado a ser operativa a escala nanométrica, lo que permite alterar y modificar la estructura molecular de algunos materiales y producir nuevos nanomateriales. Esto vale para la materia inerte y para la materia orgánica. En particular, es posible operar a escala nanométrica con el ribosoma, dado que se trata de una estructura relativamente grande a dicha escala, de unos 20 nanómetros ${ }^{16}$. Filosóficamente hablando, los nanoscopios y el resto del instrumental NTC han aumentado la capacidad de acción de los seres humanos, puesto que ahora es posible ver, observar y operar con diversos nano-objetos que ya existían en la naturaleza, pero que nunca habian podido ser manipulados por los seres humanos ${ }^{17}$. Además, es posible sintetizar nuevas estructuras atómicas, no existentes en el mundo físico y, por así decirlo, artificializar la naturaleza a escala nanométrica, con todas las repercusiones que ello tiene en los microcosmos y en los macrocosmos. La ingeniería ha expandido su campo de acción a un nuevo mundo y ha logrado construir nanomateriales, a base de modificar la estructura molecular de algunas sustancias. Esta capacidad de transformar la materia y de generar nuevas estructuras materiales que, siendo parecidas a las previamente existentes, difieren en algunos aspectos de ellas, es la gran 
fuerza de las NTC. Los nanocosmos primero habian sido imaginados y luego fueron concebidos científicamente: la teoria atómica, la mecánica cuántica y la física de partículas son buenos ejemplos de ello. La gran novedad consiste en que, además de ser concebidos e investigados conceptualmente, las NTC permiten desarrollar acciones técnicas de cambio y transformación de los diversos nanosistemas y nano-objetos. Por eso son tecnociencias, no sólo ciencias. Los nanocosmos no sólo son accesibles a la teoría, sino ante todo a la acción transformadora del ser humano. El proyecto baconiano (conocer la naturaleza para mejor poder dominarla) ha alcanzado un nuevo hito a finales del siglo $X X$, al extenderse la colonización de la biosfera y de los microcosmos a ámbitos mucho más pequeños, y hasta hace poco completamente invisibles, no manipulables y, si se quiere, vírgenes: los nanocosmos.

Las propiedades de los elementos de materia a escala nanométrica no son las mismas que a escala micro- o mesocósmica ${ }^{18}$. Por esa razón, las NTC están generando mucho conocimiento científico. Sin embargo, el interés por esta línea de investigación no se ha centrado tanto en los nuevos conocimientos que suscita sino, ante todo, en la posibilidad de transformar y modificar la estructura y las propiedades de diversos materiales: aceros más duros pero menos pesados, nuevos conductores de la electricidad y la información (fotones), medicinas que puedan atacar a los virus y las bacterias infiltrándose en su estructura molecular y modificándola, etc. En los últimos años, proliferan las noticias sobre la fabricación de nuevos materiales, que se obtienen a base de modificar la estructura molecular o celular de diversas sustancias orgánicas e inorgánicas.

La novedad estriba en que, siguiendo el ejemplo de la ingeniería genética, los materiales nanotecnológicamente modificados pueden ser patentados por las empresas tecnocientíficas que los producen. No sólo estamos ante la posibilidad de fabricar materia artificial, sino también de apropiársela con el fin de obtener beneficios mediante su comercialización ulterior. Por eso hablamos de colonización de los nanocosmos. Las expectativas de obtener beneficios económicos por parte de las empresas tecnocientíficas son muy grandes, lo que ha dado lugar a una dura competencia por tomar posiciones en el nanomundo. No se trata ya de apropiarse de territorios (nivel mesocósmico), sino de patentar nuevas formas de materia artificialmente producidas. La apropiación del conocimiento NTC puede ser altamente rentable, y no sólo desde el punto de vista económico, sino también, por ejemplo, desde una perspectiva militar. Es posible producir una nueva modalidad de armas, tanto para el ataque como para la defensa. Lógicamente, la industria militar se ha interesado en el sector NTC, como muestra la National Nanotechnology Initiative norteamericana (2000), aprobada por el Senado como una iniciativa estratégica para los EEUU y con una componente militar importante ${ }^{19}$. Desde que se probó que también era posible modificar la estructura de la materia orgánica con herramientas NTC (2000), las empresas de biotecnología se volcaron hacia el sector nano, siguiendo el ejemplo de las empresas del sector TIC, que ya han logrado producir chips a escala nanométrica e investigan sobre las posibilidades de producir y comercializar nanosensores, nanodetectores, nanotransmisores, etc. Lo que hace cuarenta años parecia ciencia ficción, y de hecho era abordado desde una perspectiva así, a principios del siglo XXI comienza a ser realidad, atrayendo a los diversos agentes que operan en los sistemas tecnocientificos de $1+D+i$. No cabe duda de que las NTC están mostrando una alta capacidad de innovación. Es cierto que hay que realizar cuantiosas inversiones para crear Centros de Investigación en nanotecnologías. Pero esas inversiones privadas pueden ser rentables a medio plazo y, si no, siempre se puede demandar que los gobiernos promuevan esas infraestructuras con cargo a fondos públicos.

En este contexto surgen los programas Converging Technologies, tanto en EUUU como en la Unión Europea, Canadá, Israel, Rusia, China y otros muchos paises. Todos los paises tecnocientíficamente desarrollados, y buena parte de los que están en vías de desarrollo, se han lanzado a la conquista y colonización de los nanomundos, siguiendo el lema de Feynman: en lo más pequeño hay sitio. Ello no da lugar a contiendas por el territorio, sino por el conocimiento y la tecnología NTC. La pugna competitiva tiene lugar en el espacio de investigación, que ha dejado de ser el feudo de las comunidades científicas para convertirse en un campo de contienda tecnocientífica en el que interviene una gran diversidad de agentes. Lo importante es tener en cuenta que, conforme se colabora y se compite por la obtención de conocimiento y tecnología NTC, se está luchando por un nuevo ámbito espacial, que tiene un tamaño mínimo, pero gran incidencia ulterior en los microcosmos y mesocosmos, al posibilitar la ingeniería de la materia, es decir, la generación de nuevas estructuras materiales, algunas de las cuales son estables y ensamblables a mayor escala. Esta idea aparece expresada con bastante claridad al comienzo del informe europeo CTEKS:

ARBOR CLXXXI 715 SEPTIEMBRE-OCTUBRE (2005) 301-315 ISSN: 0210-1963 
"CTs will form an invisible technical infrastructure for human action - analogous to the visible infrastructure provided by buildings and cities»" ${ }^{20}$.

Prolongando esa metáfora arquitectónica, cabe decir que los nanocosmos son los cimientos de cualquier estructura material, sea ésta orgánica o inorgánica. En la medida en que las NTC permiten intervenir a escala nanométrica, los edificios, las ciudades, los objetos materiales $y_{1}$ en particular, la materia viva, puede ser construida a partir de los nuevos nanomateriales generados por las NTC. En principio, esta nueva capacidad tecnocientífica afecta a los más diversos ámbitos: los materiales, la energía, las comunicaciones, el almacenamiento y procesamiento de la información, la medicina, la farmacología, los alimentos, etc. Los nanocosmos son el cimiento de la realidad. La posibilidad de colonizarlos, aunque sea parcialmente, supone un salto cualitativo del homo faber, al abrir la posibilidad de fabricar nuevas modalidades de materia viva o inerte.

Sin embargo, no todo es posible a escala nanométrica. Las leyes de la mecánica cuántica limitan las capacidades de investigación y, por así decirlo, de nanoacción. No es posible manipular a una escala de $10-12 \mathrm{~mm}$ porque las particulas se convierten en ondas y el principio de indeterminación de Schrödinger tiene validez. Sin embargo, en el intervalo de los 10-9 $\mathrm{mm}$ "queda mucho sition, como dijo Feynman, y no sólo por explorar, sino ante todo por conquistar y dominar. Las NTC tienen una fuerte base cientifica, pero también tecnológica. La dualidad onda/corpúsculo, por ejemplo, es básica en el funcionamiento de varios nanoscopios. Un segundo ejemplo es la computación cuántica, hoy por hoy inviable, pero que de lograrse transformaría radicalmente el sistema tecnológico TIC. De hecho, la investigación cientifica ya ha mostrado que, además de los electrones, también los fotones sirven para transmitir información, en este caso a la velocidad de la luz. La nanofotónica es una de las vías de investigación más prometedoras, aparte de los nanomateriales o los nanofármacos. En sus diversas direcciones y aplicaciones, los programas CT pretenden dominar los nanocosmos, con la consiguiente transformación de microcosmos y macrocosmos.

Las ciencias y las tecnologías modernas posibilitaron la emergencia y el desarrollo de la industrialización, que modificó radicalmente las sociedades, el paisaje y la riqueza de las naciones. Para ello se intervenía a escala microcósmica ductos farmacéuticos. Pues bien, las NTC permiten operar en ámbitos mucho más pequeños, al visualizar y modificar las estructuras atómicas y moleculares de las diversas formas de materia. No es de extrañar que se piense en que estamos en los albores de una nueva revolución tecnocientífica, basada en el conocimiento y la manipulación de la materia y la energía a escala nanométrica.

Para llevar a cabo y, en su caso, controlar la conquista de los nanocosmos no bastan las políticas de Estado. Los Gobiernos fomentan activamente estas líneas de investigación, a través de iniciativas de política científica como los programas Converging Technologies, pero quienes avanzan en el conocimiento y dominio de los nanocosmos son otro tipo de agentes, las empresas tecnocientíficas, siguiendo sus propias estrategias empresariales. Buena parte de ellas son empresas transnacionales privadas. Otras son consorcios de laboratorios públicos y empresas privadas, tanto a nivel nacional como transnacional. Por tanto, a diferencia de la colonización de la era industrial, no se trata de ampliar las fronteras territoriales en nombre de un pais o un Estado, llegando a acuerdos de delimitación del territorio con los Estados rivales, si hace falta. Los nanocosmos tienen una estructura métrica y topológica muy distinta a la de los mesocosmos donde se han constituido las naciones y los Estados. Por los nanomundos actuales pulula una multitud de agencias tecnocientíficas, muchas de ellas con estrategias propias de acción y de negocio. No se trata de conquistar y controlar zonas geográficas, como en la época del colonialismo industrial, sino de ganar ámbitos del conocimiento. Esta es una de las razones por las que el programa CT europeo está claramente orientado a la sociedad del conocimiento. Todo ello aporta problemas nuevos a la gobernanza de la ciencia y la tecnología, como veremos a continuación. Anticipamos uno: la propiedad del conocimiento NTC se convierte en una cuestión estratégica para la gobernanza, sobre todo si se pretende mantener la existencia de lo público en los ámbitos nanocósmicos.

\section{Los programas Converging Technologies (CT) en EEUU y la UE}

Las tecnociencias no sólo pretenden conocer mejor el mundo (observarlo, analizarlo, explicar y predecir fenómenos y sucesos...), como era el objetivo de la ciencia moderna. Además, tienden a transformarlo. El programa CT norteamericano lo anuncia claramente en su mismo título: Converging 
Technologies for Improving Human Performance. Interesa saber cómo es el mundo a escala nanométrica y para ello se requiere mucha investigación básica. Sin embargo, el fin último no es el conocimiento, sino la mejora de las capacidades de acción humana:

"Converging technologies could achieve a tremendous improvement on human abilities, societal outcomes, the nation's productivity, and the quality of life ${ }^{21}$.

El programa estadounidense NBIC pretende incrementar las capacidades humanas (perceptivas, cognitivas, comunicativas...), asi como la productividad y competitividad de las empresas. Entre los avances y ventajas competitivas que se podrían lograr, los editores del informe de la National Science Foundation, Roco y Bainbridge, mencionan las siguientes:

"improving work efficiency and learning, enhancing individual sensory and cognitive capabilities, revolutionary changes in healthcare, improving both individual and group creativity, highly effective communication techniques including brain-tobrain interaction, perfecting human-machine interfaces including neuromorphic engineering, sustainable and "intelligent" environments including neuro-ergonomics, enhancing human capabilities for defense purposes, reaching sustainable development using NBIC tools, and ameliorating the physical and cognitive decline that is common to the aging mind ${ }^{22}$.

Como puede verse, en ningún momento se pone el acento en el avance del conocimiento, aun siendo esto necesario, sino en mejorar diversas capacidades humanas, tanto individuales como colectivas. El informe CTIHP de la NSF, que suele ser denominado programa NBIC (Nano-bio-info-cogno ${ }^{23}$ ), no sólo pretende generar nuevas teorias científicas, sino ante todo transformar el mundo. Por eso habla de una unueva revolución industrialn, e incluso de un nuevo Renacimiento ${ }^{24}$. Cabe decir que el conocimiento cientifico deja de ser un fin y se convierte en un medio. En el caso de las NTC se trata de modificar las propiedades básicas de la materia (cohesión, peso, dureza, conductividad eléctrica, absorción luminosa, etc.) y por ello es una ingeniería de materiales basada en las distintas propiedades que la materia muestra a escala nanométrica ${ }^{25}$.

Ahora bien, si se trata de mejorar las performances humanas, se suscita inexorablemente un problema de valores. ¿Qué es mejor para los seres humanos? Tanto el informe europeo como el norteamericano afirman valores a fomentar y disvalores a combatir ${ }^{26}$. La novedad estriba en que la búsqueda del conocimiento ya no es el valor-fin sino, por decirlo en términos de Max Weber, un valor-medio. Los programas CT están guiados por una pluralidad de valores. Unos son epistémicos e internos a la ciencia, otros no. Hay valores tecnológicos, económicos, políticos, militares, sociales, ecológicos, jurídicos y morales que tienen su peso especifico en los programas CT. Conjugar esos valores y gestionar los conflictos que generan constituye uno de los problemas principales de la gobernanza de las nanotecnologías.

Por otra parte, existe un objetivo general, típico de los sistemas de I+D+i y de las tecnociencias: innovar. Las innovaciones que el programa norteamericano NBIC promueve tienen como destinatario último a los gobiernos (defensa, administración), los mercados (eficiencia, productividad), la nación (liderazgo mundial), la sociedad (mejora de diversos servicios) y las personas (mejores capacidades sensoriales y cognitivas, comunicación directa entre cerebros, prolongar la vida, atemperar la decadencia fisica y mental, etc.). Se investiga cómo es el nanomundo con el fin primordial de transformarlo y mejorarlo. El programa NBIC está diseñado para cambiar radicalmente y a nivel global los mercados y sociedades, introduciendo innovaciones que sean altamente competitivas y que resulten aceptables para los clientes y consumidores. En último término, se pretende modificar los hábitos y los comportamientos de las personas, de modo que incorporen a su vida cotidiana las diversas innovaciones que las nanotecnologías vayan generando.

El macroprograma europeo también tiende a una profunda transformación, pero de distinto tipo. Ha sido denominado "Converging Technologies for the European Knowledge Societyn (CTEKS) ${ }^{27}$, con lo que se señala el principal objetivo a lograr al impulsar la convergencia tecnológica: construir una sociedad europea del conocimiento que, conforme a la estrategia e-Europe 2000, convierta a la Unión Europea en el líder mundial del conocimiento en el año 2010. Independientemente de la diferencia de objetivos finales, la UE y los EEUU comparten la tesis básica: hay que innovar. En el documento titulado "Hacia una estrategia europea para las nanotecnologias" ${ }^{28}$, se dice taxativamente que "la excelencia europea en el ámbito de las nanociencias debe, finalmente, traducirse en productos y procesos comercialmente viables» ${ }^{29}$.

Pese a que la innovación es la prioridad, los documentos europeos insisten mucho más que los norteamericanos en la necesidad de investigar los riesgos:

ARBOR CLXXXI 715 SEPTIEMBRE-OCTUBRE (2005) 301-315 ISSN: 0210-1963 
"La nanotecnología se ha de desarrollar de forma segura y responsable. Su avance deberá respetar principios éticos y será preciso estudiar científicamente sus riesgos potenciales para la salud, la seguridad y el medio ambiente con el fin de prever la normativa necesaria. Habrá que evaluar y tener en cuenta el impacto a nivel social, ${ }^{30}$.

Por tanto, no sólo hay que investigar las posibilidades abiertas por las NTC, sino también sus riesgos ${ }^{31}$. Por otra parte, el programa europeo no prioriza las aplicaciones militares, a diferencia del norteamericano. La recomendación undécima del grupo de 25 expertos que preparó el plan europeo CTEKS afirma explícitamente que:

"a strict line (should) be maintained between military ambitions for CTs and their development in Europe»32.

Con esta opción estratégica, la UE optó por una convergencia tecnológica orientada a la sociedad civil, dejando de lado los desarrollos militares, muy presentes en el plan norteamericano. Conforme a la estrategia de la agenda de Lisboa (2000), que orientó a la UE hacia una sociedad europea del conocimiento, el informe CTEKS se distanció desde el principio del NBIC norteamericano, asi como de un documento previo estadounidense titulado "Converging technologies for battlefield domination» ${ }^{33}$.

Hay otras muchas diferencias entre ambos programas, que examinaremos con mayor detalle en el apartado siguiente. Esta primera aproximación, sin embargo, nos permite apreciar dos puntos comunes muy importantes:

1): Ambos programas se proponen intervenir en múltiples ámbitos científicos y tecnológicos. Otros paises acotaron más el ámbito de sus planes de convergencia tecnológica, por ejemplo Canadá, cuya estrategia de investigación se orientó fundamentalmente hacia la biología y la medicina.

2): Tanto el programa europeo como el norteamericano fueron elaborados por expertos nombrados para ese cometido, con una misión previamente establecida por las autoridades respectivas de politica científica. En ambos casos, sin embargo, dichas autoridades habian recibido la demanda previa de diseñar programas de convergencia tecnológica por parte de diversos agentes tecnocientíficos, del ámbito privado y del público. Además, algunos países europeos y algunos Estados de la Unión norteamericana ya habian emprendido inicia- tivas por sí mismos, en particular en el campo de las nanotecnologias. Por tanto, los NBIC y CTEKS son acciones de gobernanza que:

a): recogen demandas previas de agentes tecnocientíficos influyentes,

b): son promovidas por departamentos gubernamentales de política científica y tecnológica,

c): están diseñadas por grupos de expertos que han recibido un encargo por parte de las autoridades de política científica,

d): los grupos de expertos tratan de aglutinar en un único plan de acción las diversas demandas e iniciativas previas, orientándolas en base a unos objetivos que confluyen con las estrategias políticas, sociales y económicas de las autoridades que les han encargado la misión de elaborar dichos informes,

e): los informes son aprobados, presupuestados e implementados ulteriormente mediante programas específicos que han de ser aprobados por los Parlamentos y los Gobiernos y Comisiones,

f): a partir de todo lo anterior, comienza a producirse la convergencia tecnológica propiamente dicha, y ello durante un lapso de tiempo prolongado ${ }^{34}$,

g): el desarrollo de los programas CT es evaluado periódicamente, y no sólo para valorar el avance en el conocimiento científico o los desarrollos tecnológicos generados, sino también las innovaciones resultantes de dichos programas, así como su recepción social.

h): Este seguimiento y evaluación de los grandes programas de investigación es otra de las caracteristicas de la tecnociencia, en particular en los paises donde hay sistemas de $\mathrm{I}+\mathrm{D}+\mathrm{i}$ consolidados. Ello permite volver a ponderar los diversos programas que son promovidos en función de su nivel de éxito, asi como reorientar las políticas y las inversiones en $\mathrm{I}+\mathrm{D}$.

i): Así como los rasgos anteriormente descritos son comunes a la actividad tecnocientífica contemporánea, y por ende a los programas $\mathrm{CT}$, tanto el programa NBIC norteamericano como el CTEKS europeo coinciden en otro rasgo, que resulta bastante singular y tiene pocos precedentes en la reciente historia de la tecnociencia: reorganizar las comunidades académicas y las empresas que se dedican a la investigación y el desarrollo promoviendo una 
convergencia disciplinar, interinstitucional e intersectorial. Surge asi un nuevo problema para la gobernanza, de gran interés, aunque en este artículo no vayamos a ocuparnos de la reorganización de los espacios de investigación y enseñanza superior que comporta el desarrollo de los programas CT.

Otros muchos paises están impulsando iniciativas similares: Japón, Corea, Taiwán, China, Rusia, Australia, Canadá, India, Israel, algunos paises latinoamericanos, Nueva Zelanda, Filipinas, Singapur, Sudáfrica, Tailandia, etc. Las nanotecnologías conforman una cuestión de gobernanza global, no sólo nacional, como ya dijimos al principio. Cada pais tiene sus propias estrategias. Otro tanto cabe decir de las empresas transnacionales que fomentan la investigación, el desarrollo y la innovación. Aunque en este artículo tampoco vayamos a ocuparnos de los demás programas $\mathrm{CT}$, centrándonos únicamente en los programas europeo y norteamericano, si queremos subrayar el carácter transnacional de este tipo de iniciativas. Los estudios sobre gobernanza deben incorporar una metodología comparativa, y no sólo entre paises, sino también entre las diversas agencias tecnocientíficas, sean públicas, privadas o mixtas, grandes o pequeñas.

\section{Agentes y principios-guia que intervienen en el diseño de los programas CT}

A diferencia de los EEUU, donde las Agencias militares de investigación fueron unos de los principales promotores del programa NBIC, el proyecto europeo, que surgió a rebufo del norteamericano, fue elaborado fundamentalmente por académicos. De los 25 expertos europeos reunidos en Oslo, la presidenta del grupo era historiadora y el relator filósofo. Luego habia expertos en biotecnologias, nanotecnologías, tecnologías de la información y ciencias cognitivas, asi como economistas y teóricos de la innovación, historiadores y filósofos de la ciencia y la tecnología, éticos, juristas y especialistas en ciencias sociales, prospectiva, educación, evaluación de tecnologias y política científica. Llama la atención, por ejemplo, que entre los 25 expertos europeos hubiera cuatro que trabajan en Departamentos de Filosofia (Nordmann, Andler, Dupuy y Rip), sobre todo si se compara esa composición con los redactores del informe norteamericano.

El documento norteamericano lo editaron un fisico (Roco) y un sociólogo (Bainbridge), pero fue elaborado por 32 repre- sentantes del Gobierno y de los Laboratorios Nacionales, 29 académicos y 19 representantes del sector privado. De los primeros, 7 personas provenian de la National Science Foundation, entre ellos los dos editores del informe NBIC, 5 de la NASA, 3 de los Laboratorios Sandia, uno de la Executive Office of the President, dos del Departamento de Defensa, uno de la Navy, uno de las Fuerzas Aéreas, cinco de diversos Departamentos del Gobierno o la Cámara de Representantes en Washington, uno de la Comisión Reguladora de la Energía Nuclear, dos del Departamento de Comercio, uno del de Energía, uno de la Oficina de Patentes, etc. El grupo europeo no contaba con este tipo de representantes, dos a lo sumo, y apenas dos o tres provenientes del sector privado. Composiciones tan diferentes dan una primera idea de la diversidad de agentes que diseñaron el programa norteamericano y el europeo. En el caso europeo, los miembros del grupo de expertos fueron propuestos por los diversos paises de la UE, los cuales optaron mayoritariamente por académicos. En el caso norteamericano, los académicos eran minoria y la presencia del Gobierno a través de diversos Departamentos e instituciones, asi como la de las empresas privadas, resultó muy significativa.

Partiendo de estas diferentes sensibilidades a la hora de componer los grupos de expertos, eran de esperar importantes diferencias entre ambos proyectos, como efectivamente ocurrió. Pero, además, el grupo europeo estaba sujeto a un mandato previo, que incluia hasta diez principios-guia a los que debían ajustarse los miembros del grupo. Dicho documento, el Group Mandate, habia sido elaborado previamente por la Science and Technology Foresight Unit de la Unión Europea, y tiene un gran interés desde el punto de vista de la gobernanza. Aqui comentaremos únicamente los siguientes puntos:

a): El documento europeo tiene como referencia el informe de la NSF Norteamericana, el cual, sin embargo, no debería constituir el "punto focal de la reflexión»35. La segunda pregunta que se hace al grupo de expertos en relación a las CT es la siguiente: "ls there a European vision?». Por tanto, se trataba de dilucidar si Europa podía aportar un plan propio y diferente del norteamericano que contribuyera a consolidar la Unión Europea, no sólo a generar conocimiento científico y desarrollos tecnológicos. Esta es la razón principal por la que el grupo europeo de expertos adoptó una perspectiva más orientada a la sociedad que el norteamericano, y en concreto hacia la sociedad europea del conocimiento, conforme a la Agenda previa de Lisboa 2000. 
b): El Mandato al grupo europeo insistía en que "it is a priority to clarify the civil and societal benefits of this research to give them a new legitimacy and to put them firmly in a context of positive social dynamicsi" ${ }^{36}$. Por tanto, el grupo de expertos tenia que preocuparse por legitimar socialmente el programa CT. Llegaba a afirmarse que el principio de precaución debería ser tenido en cuenta a la hora de fijar el marco de la investigación en el ámbito $\mathrm{CT}$, algo impensable en el informe norteamericano.

c): La tercera pregunta versaba sobre el papel de las ciencias sociales en el impulso a las tecnologías convergentes, y la cuarta subrayaba el papel de las ciencias cognitivas, otra de las especificidades del proyecto europeo frente al norteamericano. La sexta y última cuestión aludía al impacto educativo, que si había sido suscitado en el informe de la NSF. Por tanto, ambos planes coinciden en algunas áreas prioritarias, pero difieren en otras, siempre en función de sus respectivas estrategias generales, que son de indole política, económica y social. La investigación propiamente científica está subordinada a esas estrategias, como Muñoz ha señalado en su definición de "gobernanza".

d): El Mandato europeo indicaba tres objetivos, el primero de los cuales comportaba una clara contraposición con el programa NBIC norteamericano: "the objective being to show how this approach can break with the idea of the individual performance being the only criteria for measuring successin ${ }^{37}$. Por tanto, el proyecto europeo pretendía contraponerse desde el principio al norteamericano, al poner el acento en los beneficios sociales que puede aportar la convergencia de tecnologias, más que en la pura implementación de las capacidades individuales mediante tecnologías nano-bioinfo-cogno.

e): Además de los objetivos, el Mandato de la UE explicitaba cinco preguntas básicas a las que el grupo de expertos debia responder. Llama la atención la tercera. Sobre todo si se compara el documento final europeo con el norteamericano: "What are the ethical and societal concrete questions that the implementation of the technologies concerned raised? ${ }^{38}$. Siendo ésta una de las cuestiones, y dados los objetivos y el marco previo, la composición del grupo europeo de expertos se entiende bien, a diferencia del grupo norteamericano, para el que los problemas éticos y sociales, aun existiendo, no eran una prioridad a la hora de diseñar el programa $\mathrm{NBIC}^{39}$. f): Por último, entre los principios-guía del Mandato europeo hay dos que señalan una significativa preocupación por los problemas ecológicos que pudieran derivarse de los programas CT: el séptimo (sostenibilidad) y el noveno (principio de precaución, anticipación y gestión de los riesgos). No es de extrañar que, una vez finalizado el informe CTEKS, se recomendara investigar los riesgos que pueden derivarse de la convergencia tecnológica, y en particular de las NTC. Esta es otra de las grandes diferencias entre ambos programas.

Habria otros muchos aspectos a comentar a la hora de señalar las diferencias entre el informe norteamericano y el europeo. Sin embargo, los seis anteriores pueden bastar como primera aproximación. Aun teniendo rasgos comunes, ambos documentos muestran la contraposición entre dos modelos de gobernanza de la tecnociencia, uno mucho más orientado a lo individual, el sector privado y las aplicaciones militares, y otro que tiende más a satisfacer objetivos sociales, del sector público (consolidación y ampliación de la propia UE) y a las aplicaciones civiles. Un análisis más detallado permitiria caracterizar mejor ambos modelos de gobernanza. Para ello habría que estudiar las implementaciones y desarrollos ulteriores de los programas NBIC y CTEKS en forma de acciones concretas de política cientifica. En cualquier caso, y aunque sólo nos hayamos centrado en el diseño de dichas políticas, podemos concluir que tanto la fase de diseño como los agentes concretos que son seleccionados para ello y los mandatos o marcos previos en los que dichas acciones de diseño se llevan a cabo tienen una gran importancia a la hora de definir diversos modelos de gobernanza de las tecnociencias. Dicho de otra manera: la gobernanza es una acción estratégica ex ante, basada en principios, valores e intereses generales que se trata de fomentar al poner en marcha estos grandes programas de investigación. La participación de diversos agentes en esa fase previa de diseño, cada uno de los cuales encarna diferentes sensibilidades, intereses y valores, es otra de las caracteristicas de la gobernanza de las tecnociencias. Frente al modelo lineal de Vannevar Bush (1945), según el cual había que impulsar incondicionalmente el avance del conocimiento científico en régimen de estricta libertad para las comunidades investigadoras, la gobernanza de las tecnociencias sigue estrategias multilineales, al involucrar a una pluralidad de agentes, intereses y valores que han de aglutinarse y armonizarse desde la fase de diseño de los grandes programas tecnocientíficos. El caso de las nanotecnologias y su progresiva integración en los programas 
Converging Technologies muestra claramente que de la politica cientifica del siglo XX a la gobernanza de la tecnociencia del siglo XXI se han producido profundos cambios estructurales en la práctica investigadora.

\section{Pluralidad de agendas cientificas}

Para terminar, mostraremos la importancia que tiene la noción de agenda tecnocientífica a la hora de analizar la gobernanza contemporánea de la ciencia y la tecnologia.

Dicha noción es utilizada una y otra vez al diseñar los programas CT, en particular en el caso europeo. Por esta razón, nos centraremos en el documento CTEKS. Sin embargo, asi como la noción de gobernanza es difusa y polívoca en su uso actual, también se requiere un esfuerzo de elucidación del concepto de agenda científica, que debería ser introducido como una noción técnica en los estudios sobre gobernanza contemporánea.

Desde el principio, el informe CTEKS alude a diversas agendas científicas en relación a los programas CT. Se trata de satisfacer una demanda de la Unión Europea, puesto que el Mandato formulado por la Science and Technology Foresight Unit de la UE en 2003 dejaba claro que el primer objetivo que debía lograr el grupo de expertos consistía en idevelop a research agenda focused on social sciences research on co-operation modelsı de dimensión especificamente europea ${ }^{40}$. Por tanto, la opción estratégica de orientar el programa CT hacia la sociedad europea del conocimiento no sólo se adecuaba a la Agenda de Lisboa 2000, sino que promovía la cooperación entre los investigadores europeos. La investigación europea en nanotecnologias, al igual que el programa CTEKS, está orientada a la consecución de objetivos de mayor calado, tales como la creación de un espacio europeo de investigación y el desarrollo de una sociedad europea del conocimiento. La orientación social del programa CTEKS no viene marcada por el interés científico que puedan tener las ciencias sociales, sino por tendencias estratégicas más generales a las que debe adecuarse la investigación científica. Dicho de otra manera, los objetivos científicos y tecnológicos en el ámbito NTC son importantes, pero están claramente subordinados a metas más complejas, de indole politica, social y en parte económica, puesto que la Agenda Lisboa 2000 propugna la competitividad de la UE en relación a los EEUU en un ámbito especifico, que no es militar sino civil: la sociedad (global) del conocimiento. La convergencia tecnológica nano-bio-info- cogno, junto con la colaboración de otras áreas disciplinarias (Ciencias Sociales, Antropologia, Derecho, Ética, incluso Filosofia), puede favorecer la investigación, como había postulado el informe norteamericano de 2001, pero, sobre todo, potencia la propia convergencia europea, al favorecer la creación de un espacio común de investigación científica, hasta entonces muy poco estructurado. El objetivo real del programa CTEKS es la creación del espacio europeo de investigación, más que el avance del conocimiento científico. La creación de consorcios y redes europeas en los ámbitos $\mathrm{CT}$, e incluso la cooperación con paises emergentes (se menciona a China y la India), definen la agenda tecnocientífica europea, que se plasma en el informe CTEKS, y ulteriormente en las acciones de la Comisión para promover dicho plan, incluido el VII Programa Marco.

Podemos concluir, por tanto, que una agenda tecnocientífica no se circunscribe a los aspectos científicos o tecnológicos de un programa de investigación, sino que incluye otro tipo de objetivos, de índole muy diferente. Eso también ocurre en el programa norteamericano NBIC, aunque esta agenda sea muy distinta a la europea. Las diferencias que hemos constatado entre ambos informes dependen en último término de las agendas tecnocientíficas contrapuestas que mantienen los EEUU y la UE en la primera década del siglo XXI. Pero en uno y otro caso, los proyectos y programas de investigación se desarrollan en el marco de agendas tecnocientíficas. Estas son el problema central para la gobernanza.

El informe CTEKS europeo menciona varias veces las diversas agendas que pueden ponerse en marcha para desarroIlar un programa CT: la norteamericana, la canadiense, la europea y otras muchas posibles. Según sus autores, pueden impulsarse programas CT para:

- mejorar las capacidades humanas (informe NSF)

- para dominar el campo de batalla (propuestas de sectores militares estadounidenses)

- para desarrollar una sociedad del conocimiento (proyecto europeo)

- para producir una sintesis de los biosistemas (proyecto canadiense)

- para la acuicultura, la alimentación y el medio ambiente (proyecto noruego)

- para el procesamiento de los lenguajes naturales (ejemplo del grupo europeo)

- para el desarrollo de la inteligencia artificial (segundo ejemplo posible del grupo europeo de expertos)

ARBOR CLXXXI 715 SEPTIEMBRE-OCTUBRE (2005) 301-315 ISSN: 0210-1963 
- para objetivos más específicos: tratamiento de la obesidad, manos artificiales (red europea Nano2Life), etc. - para lograr otro tipo de objetivos.

Al contraponer esa pluralidad de agendas tecnocientíficas posibles para impulsar y diseñar un programa $\mathrm{CT}$, los expertos del grupo europeo extrajeron una conclusión que tiene relevancia filosófica, porque permite caracterizar en parte la noción de agenda tecnocientifica. Tras afirmar que las NTC son tecnologias capacitadoras (enabling technologies) para las TIC y las biotecnologias, postularon que también las ciencias sociales y las humanidades pueden ser capacitadoras para diversos sistemas tecnológicos, al orientar las agendas tecnocientíficas correspondientes en una dirección social y humanística. Esta fue la opción estratégica que eligieron, siguiendo el Mandato de la $U E$, que les preguntaba por el papel de las ciencias sociales a la hora de desarrollar programas CT en Europa. De lo contrario -advirtió el grupo de expertos- "without an understanding of society, it is all too easy for a technology to be launched improperly and subsequently rejected by society" ${ }^{41}$.

Aparece así un nuevo agente en la gobernanza de las tecnociencias, las sociedades, que deben ser muy tenidas en cuenta a la hora de impulsar programas de la envergadura de los que estamos comentando. El grupo de expertos europeos orientó el programa CT en una dirección social porque asumió que el éxito de los desarrollos tecnológicos y de las innovaciones depende de las sociedades, en último término. En la medida en que una revolución tecnocientífica satisfaga los intereses de una sociedad y sea bien recibida por ésta, sus posibilidades de éxito son mayores. Habiendo descubierto que en las diversas agendas CT hay un patrón común a todas sus formulaciones, CT para ..., el problema de la gobernanza se centra en las metas y objetivos que orientan los programas $\mathrm{CT}$, no simplemente en el avance del conocimiento, los desarrollos tecnológicos y las innovaciones. Las grandes acciones de gobernanza (agendas tecnocientificas) deben tener muy claros sus fines, y evaluarlos adecuadamente. En el fondo, el conocimiento científico y la tecnología son medios para alcanzar esos fines (militares, sociales, sanitarios, empresariales, etc.), no fines en sí mismos. Por eso dijimos al principio de este artículo que en la gobernanza contemporánea los avances en el conocimiento son valores-medios, en la terminología de Weber, no valoresfines, como ocurria en la ciencia moderna.

Desde una perspectiva ontológica, podriamos decir que los programas CT son funciones no saturadas, en el sentido de
Frege. Los objetivos y metas de dichos programas dan contenido a las diversas agendas tecnocientíficas. Aunque aquí no vayamos a profundizar en estas cuestiones ontológicas, si señalaremos que, parafraseando al grupo europeo, las acciones tecnocientificas del tipo CT pueden ser representadas formalmente de la manera siguiente: CT para $X$ donde $X$ define los diversos tipos de objetivos que unos $u$ otros agentes tecnocientíficos (gobiernos, empresas, centros de investigación, etc.) pueden tener a la hora de impulsar los programas CT o participar en ellos.

Otro tanto cabría decir de las agendas tecnocientíficas en general, no sólo de los programas CT. Una agenda tecnocientífica determinada está orientada y guiada por un conjunto de objetivos, metas y valores, que pueden ser de muy diversa indole según los diversos agentes. Si ello es así, el principal problema de la gobernanza NTC consiste en integrar y priorizar esa pluralidad de objetivos y valores, que no sólo son científicos y tecnológicos, sino también económicos, comerciales, sociales, politicos $y_{\text {, en algunos }}$ casos, militares. En el marco de una misma agenda tecnocientífica opera una pluralidad de agentes con intereses y objetivos parcialmente concordantes, pero también parcialmente divergentes y contrapuestos. La gobernanza de la tecnociencia interviene en un espacio plural, rompiéndose con ello el modelo lineal de Vannevar Bush. De ser el fin máximo, como se decía en el informe de 1945, Science: the Endless Frontier, el conocimiento cientifico deviene un medio para lograr objetivos económica-, empresarial-, militar- o socialmente importantes. A título de conclusión, tal es la diferencia principal entre la gobernanza de los programas NTC y CT y la política científica de la ciencia moderna.

Consecuencia: el conocimiento científico deviene un instrumento para la gobernanza, al igual que los desarrollos tecnológicos. Cuando se habla de sistemas de I+D+i se está priorizando implícitamente el resultado final, la innovación, punto éste en el que coinciden los informes norteamericano y europeo. Las diferencias estriban en el tipo de innovaciones que se pretendan: militares, empresariales, juridicas o sociales, por mencionar cuatro grandes opciones posibles. Por lo general, la gobernanza contemporánea nunca opta por una única meta, sino por varias a la vez, que son satisfechas en mayor o menor grado. Este es el procedimiento básico para conjugar y armonizar los sistemas multiagentes actuales, sin perjuicio de los conflictos internos que dicha integración de intereses, valores y objetivos suscita. 
Por último, y como también advertimos al principio: la tecnociencia actual se desarrolla en un ámbito internacional (o global), razón por la cual varias agendas tecnocientíficas son impulsadas a la vez. Los paradigmas científicos son los mismos para unos y otros, por ejemplo la mecánica cuántica, pero su utilización para generar innovaciones es muy diversa. Sin perjuicio de que unos agentes tengan más peso internacional que otros (caso de la NSF o de la Comisión Europea), una gobernanza racional consiste en adecuar los medios de que dispone un determinado agente tecnocientífico a los fines y metas que pretende lograr. En lugar de competir en el terreno militar, por ejemplo, la Unión Europea decidió intentar liderar el desarrollo de la sociedad del conocimiento a nivel mundial (Lisboa 2000). Independientemente de que ese objetivo vaya a lograrse o no, cosa que resulta muy dudosa a finales de 2005 , lo cierto es que la agenda tecnocientífica europea, y en particular la agenda CTEKS, queda considerablemente determinada por esa estrategia general. Conforme a la definición mencionada al principio (véase E. Muñoz, en este mismo volumen) la gobernanza de las NTC "es la puesta en práctica de formas de gobierno estratégicas para poner de relieve el valor de lo público a través de la relación entre sociedad, mercado y Estado y conseguir de este modo un desarrollo socialmente sosteniblen. Esta definición se adecua bien al proceso de toma de decisiones que llevó a la UE a promover la investigación NTC y el programa CTEKS, así como a implementarlo mediante acciones ulteriores en el VI y VII Programa Marco de la UE. Sin embargo, otras agendas tecnocientíficas, como la norteamericana, no pretenden poner de relieve el valor de lo público, sino quizá mantener la primacía como superpotencia a nivel mundial, y paralelamente el liderazgo en el comercio y la industria internacional, como ya se decia en el informe V. Bush de 1945 y, aunque de otra manera, vuelve a repetirse en 2001.

Por ello insistimos en que los valores y principios que guian las agendas tecnocientíficas son factores muy relevantes para analizar las diversas formas de gobernanza actualmente en juego. Eso sí: todas ellas coinciden en la utilización instrumental del conocimiento científico como medio para lograr los objetivos estratégicos que los diversos agentes tienen. La emergencia de las tecnociencias se caracteriza por esta subordinación relativa de la ciencia, que se convierte en una componente más de sistemas tecnocientíficos más amplios. En el trasfondo, además, aparece un nuevo agente, la sociedad, muy presente en el informe europeo, bastante en el norteamericano.

Las relaciones entre las agendas tecnocientíficas y las sociedades serán claves para la gobernanza de la ciencia y la tecnología en el siglo XXI. 
1 Este artículo ha sido elaborado en el marco del proyecto de investigación HUM2005-02105/FISO sobre "Racionalidad axiológica de la práctica tecnocientifica: los programas Converging Technologies y su incidencia en la medicina y en la sociedad", financiado por el Ministerio de Educación y Ciencia.

2 The Royal Society \& The Royal Academy of Engineering las define de la siguiente manera: "Nanoscience is the study of phenomena and manipulation of material at atomic, molecular and macromolecular scales, where properties differ significantly from those at larger scale; Nanotechnologies are the design, characterisation, production and application of structures, devices and systems by controlling shape and size at nanometre scalen (European Industrial Research Magazine, december 2003, p. 7, disponible en www.nanotec.org.uk/report).

3 Es habitual hablar de la urevolución industrial del siglo XX|» al referirse a las NC y NT.

4 La convergencia disciplinaria que suscitan las NC y NT exige una profunda reestructuración del espacio de investigación, tanto por paises como a nivel supranacional (European Research Area, ERA). EI espacio universitario también ha de adaptarse al nuevo contexto, remodelando sus titulaciones.

5 Véase el artículo Gobernanza, ciencia, tecnología y política: trayectoria y evolución de Emilio Muñoz en este mismo volumen.

6 Esta hipótesis ha sido ampliamente desarrollada en: Echeverría, 2003.

7 Converging Technologies for Improving Human Performance (CTIHP), NSF 2001 y Converging Technologies for the European Knowledge Society, UE, 2004.

8 Feynman, 1961.

9 Taniguchi, 1974.

10 Ver E. Drexler, 1986 y 1992.

11 Raymond Bouchard, Bio-Systemics Synthesis, Canadian National Research Council, June 2003.

12 Tal fue el título de dicha conferencia. El texto fue publicado dos años después (Feynman, 1961).
13 Particular importancia tuvo el descubrimiento de los nanotubos de carbón y la construcción de modelos de realidad virtual conectados a STMs, que permitieron a los científicos "ver" y "tocar" los átomos, y a la postre operar con ellos, modificando sus posiciones relativas a escala nanométrica. Un equipo de investigadores de la Universidad de Osaka ha anunciado recientemente (Nature Materials, 2005) que ha sido capaz de mover átomos individuales sobre una superficie a temperatura ambiente. Para ello ha utilizado un nuevo tipo de microscopio, el microscopio de fuerzas atómicas (AFM). Desarrollar la capacidad de manipular los átomos es el principal objetivo de las NT, y va siendo alcanzado paso a paso.

14 Ver J. Hennig, Changes in the Design of Scanning Tunneling Microscopic Images from 1980 to 1990, en Techné 8:2 (2004).

15 Sin embargo, también las diversas representaciones que proporcionan los STM tienen interés para la gobernanza, puesto que los nanocientificos siempre se han preocupado por construir representaciones que se adecuen a las imágenes culturales previamente existentes sobre los átomos y las moléculas.

16 A escala nano, una célula es una entidad muy "grande", con un tamaño de 1 micrómetro (10-6).

17 Esta es la estrategia bottom-up, propuesta por Drexler (1986). Procesos tan básicos como la fotosintesis, indispensables para la vida de las plantas y la producción de oxígeno y energía, se producen de forma natural a escala nanométrica. Otro tanto cabe decir de otros muchos fisiológicos, incluidos los ataques de virus y bacterias.

18 Ello se debe a que, a escala nanométrica, los átomos que están en la superficie suelen tener propiedades diferentes a los que están en el centro de una partícula. Los metales, por ejemplo, devienen semiconductores 0 aislantes. Otras sustancias convierten la luz en electricidad. De ahi las enormes expectativas que las NTC han generado en el campo de la energía.

19 El MIT creó en marzo de 2002 el MIT Institute for Soldier Nanotechnology con más de 44 investigadores dedicados a desarroIlar una especie de "esqueleto exterion" (o armadura NTC) para el ejército norteamericano. Ver NSF (2001), p. 338.
20 Informe CTEKS, UE 2004, p. 3. En p. 7 se insiste en la misma idea: "an invisible technical infrastructure for human action".

21 Roco \& Bainbridge, o.c., p. IX.

22 lbid.

23 Se trata de hacer converger las nanotecnologias, biotecnologias, tecnologias de la información y ciencias cognitivas.

24 "Convergence of the sciences can initiate a new renaissancen (Roco \& Bainbridge, o.c., p. X).

25 Por ejemplo, los nanotubos de carbono son aislantes o conductores en función del diámetro del nanotubo.

26 La seguridad, por ejemplo, es un valor prioritario en ambos programas. En el informe europeo, se pretende armonizar los avances tecnocientificos NTC con el desarrollo económico que de ellos se derivan y uthe values of diversity, social justice, international security and environmental responsibility" (p. 9).

27 A. Nordmann (ed.), 2004.

28 Bruselas, 12.5.2004, $\operatorname{COM}(2004) 338$ final.

29 lbid., p. 3.

$30 \mathrm{lbid}$.

31 Ver el informe de la Unión Europea, Nanotechnologies, A Preliminary Risk Analysis, 2004.

32 CTEKS (2004), p. 9. Dicho grupo de expertos, que fue presidido por Kristine Bruland y que tuvo como relator a Alfred Nordman, se constituyó en diciembre de 2003 $y_{1}$ tras cuatro reuniones en Oslo, emitió su informe en julio de 2004.

33 Ya en julio de 1998 tuvo lugar en Cambridge, Massachusetts, la primera Nanotechnology for Soldier Systems Conference.

34 En el caso europeo, los CTEKS arrancaron con fuerza en el VI Programa Marco, y continuarán en el VII PM, pero no hay que olvidar que, como afirma otro documento de la UE, esta acción estratégica dura hasta 2020, en particular en el ámbito de las NCT.

35 CTEKS, UE, 2004, p. 61.

36 Ibid., p. 61.

37 lbid.

38 lbid., p. 62. 
39 Sin embargo, no hay que olvidar que también la NSF editó un libro sobre las implicaciones sociales del programa NBIC. Ver Roco y Bainbridge, 2001.

40 CTEKS, 2004, p. 61.

41 CTKES, 2004, p. 15.

\section{BIBLIOGRAFÍA}

Baird, D., Nordmann, A. \& Schummer, J. (eds.) (2004): Discovering the Nanoscale, Amsterdam: IOS Press.

Bouchard, R. (2003): Bio-Systemics Synthesis, Canadian National Research Council.

Bush, V. (1945): Science: the Endless Frontier, Washington: United States Government Printing.

Comisión Europea (2001): Hacia una visión estratégica de las ciencias de la vida y la biotecnología, europa.eu.int/comm/bbbiotechnology/pdf/ doc_es.pdf.

Comisión Europea (2004a): Communication from the Commission: Towards a European Strategy for Nanotechnology, COM (2004) 338, section 3.1.1., CE

Comisión Europea (2004b): Nanotechnologies: A Preliminary Risk Analysis, Bruselas: CE.

Drexler, K.E. (1986): Engines of Creation: The Coming Era of Nanotechnology, New York: Anchor Press.

Drexler, K.E. (1992): Nanosystems: Molecular Machinery, Manufacturing and Computation, New York: John Wiley.

Drucker, P. (1998): The Discipline of Innovation, Claremont: Drucker Foundation News.

Echeverría, J. (2002): Ciencia y Valores, Barcelona: Destino.

Echeverria, J. (2003): La revolución tecnocientifica, Madrid: FCE.

Feynman, R.P. (1961): There's Plenty of Room at the Bottom, en H. D. GILBERT (ed.), Miniaturization, New Cork: Reinhold Publ. Corp., 282-296.

Gardner, J.W. \& Hingle, H.T. (1999): From instrumentation to nanotechnology, Gordon \& Breach Publishing Group.

Hennig, J. (2004): Changes in the Design of Scanning Tunneling Microscopic Images from 1980 to 1990, Techné 8: 2 .

Murday, J.S. (1999): Science and technology of nanostructures in the Department of Defense, Journal of Nanoparticle Research 1: 501-505.

Nordmann, A. (ed.) (2004): Converging Technologies: Shaping the Future of the European Societies, Bruselas: UE.

Pickering, A. (ed.) (1992): Science as Practice and Culture, Chicago: University of Chicago.

Pickering, A. (1995): The Mangle of Practice, Chicago: Univ. of Chicago Press.
Roco, M.C. \& Bainbridge, W.S. (eds.) (2001a): Converging Technologies for Improving Human Performance, Virginia: NSF.

Roco, M.C. \& Bainbridge, W.S. (eds.) (2001b): Societal Implications of Nanoscience and Nanotechnology, Dordrecht: Kluwer.

Serena, P.A. \& Correia, A. (2003): Nanotecnología: el motor de la próxima revolución tecnológica, Apuntes de Ciencia y Tecnologia 9: 32-42.

Taniguchi, N. (1974): On the Basic Concept of Nanotechnology, Proceedings of the International Conference on Production Engineering, Tokio: 18-23.

The Royal Society $\&$ The Royal Academy of Engineering (2003): Report on Nanotechnologies, European Industrial Research Magazine, december 2003: 7; disponible en www.nanotec.org.uk/report.

ARBOR CLXXXI 715 SEPTIEMBRE-OCTUBRE (2005) 301-315 ISSN: 0210-1963 